\title{
Frey operation for chronic pancreatitis associated with pancreas divisum: case report and review of the literature
}

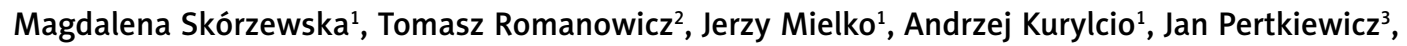 \\ Robert Zymon², Wojciech P. Polkowski ${ }^{1}$ \\ ${ }^{1}$ Department of Surgical Oncology, Medical University of Lublin, Poland \\ ${ }^{2}$ Department of General and Oncological Surgery, Pope John Paul II Regional Hospital, Zamosc, Poland \\ ${ }^{3}$ Olympus Endotherapy, Warsaw, Poland
}

Prz Gastroenterol 2014; 9 (3): 175-178

DOI: $10.5114 /$ pg.2014.43581

Key words: pancreas divisum, chronic pancreatitis, surgery.

Address for correspondence: Prof. Wojciech P. Polkowski MD, PhD, Department of Surgical Oncology, Medical University of Lublin, 11 Staszica St, 20-081 Lublin, Poland, phone: +48 8153443 13, fax: +48 8153223 95, e-mail: wojciech.polkowski@umlub.pl

\begin{abstract}
Pancreas divisum (PD) is the most common congenital anomaly of the pancreas, which increases susceptibility to recurrent pancreatitis. Usually, after failure of initial endoscopic therapies, surgical treatment combining pancreatic resection or drainage is used. The Frey procedure is used for chronic pancreatitis, but it has not been reported to be applied in an adult patient with PD-associated pancreatitis. The purpose of the paper was to describe effective treatment of this rare condition by the Frey procedure after failure of interventional endoscopic treatment. A 39-year-old female patient was initially treated for recurrent acute pancreatitis. After endoscopic diagnosis of PD, the minor duodenal papilla was incised and a plastic stent was inserted into the dorsal pancreatic duct. During the following 36 months, the patient was hospitalised several times because of recurrent episodes of pancreatitis. Thereafter, local resection of the pancreatic head combined with lateral pancreaticojejunostomy was performed with no complications. After 54 months of follow-up, the patient demonstrates abnormal glucose metabolism, with a need for enzyme supplementation, and she is free of pain. Local resection of the pancreatic head combined with lateral pancreaticojejunostomy (Frey procedure) offers a favourable outcome after failure of endoscopic papillotomy and duct stenting for pancreatitis associated with PD.
\end{abstract}

\section{Introduction}

Pancreas divisum (PD) is the most common congenital anomaly of the pancreas. The incidence has been reported to be between $5 \%$ and $11 \%$ of the population [1]. Pancreas divisum arises as a result of incomplete fusion of the dorsal and ventral ductal structures. This leads to the persistence of two drainage systems. The ventral duct (duct of Wirsung) drains only the head of the pancreas through the major papilla. The dorsal duct (duct of Santorini) drains the body and tail of the pancreas through a smaller minor papilla. Anatomical abnormalities, such as PD, increase resistance to duct secretion and could antagonise the effect of secretion-weakening cystic fibrosis transmembrane conductance regulator mutations and further increase susceptibility to recurrent pancreatitis [2].
Pancreas divisum may cause recurrent abdominal pain. Additionally, the stenosis of the minor papilla has been implicated as a cause for recurrent obstructive pancreatitis. Confirmation of the PD diagnosis is made by endoscopic retrograde cholangiopancreatography (ERCP). Pancreas divisum is found in approximately $7 \%$ of the population on ERCP, with rates ranging from $4 \%$ to $14 \%$ on autopsy series [3-5]. Endoscopic therapies of PD include papillotomy of the minor papilla with or without sphincterotomy of the major ampulla, ductal balloon dilatation and pancreatic dorsal duct stent placement. Surgical therapy includes minor papilla sphincterotomy and sphincteroplasty, but results have been suboptimal $[6,7]$. This may be due to the presence of irreversible parenchymal changes and persistence of chronic inflammation. In patients with obvious changes of chronic pancreatitis, surgical treatment should 
consist of pancreatic resection or drainage [8]. More recently, duodenum preserving pancreatic head resection was offered as an option for surgical treatment of PD causing chronic pancreatitis [9].

The Frey procedure combines a longitudinal decompression of the pancreatic duct in the body and tail of the gland, with a subtotal resection of the pancreatic head that preserves the duodenum [10]. The operation was originally described for patients with head-predominant disease, on the assumption that the enlarged complex head, full of fibrosis and obstructed ducts, was not adequately addressed by simply decompressing the main pancreatic duct by the lateral pancreaticojejunostomy alone [11]. The Frey procedure is actually applicable to a wide variety of patients with chronic pancreatitis [12].

The aim of this report is to describe effective treatment of pancreatitis associated with pancreas divisum by the Frey procedure after failure of interventional endoscopic treatment.

\section{Case report}

A 39-year-old female patient was initially treated for recurrent acute pancreatitis, which was diagnosed based on abdominal pain, hyperenzymaemia and structural pancreatic abnormalities on abdominal ultrasound and/or computed tomography (CT) scan. There was neither history of alcohol consumption nor family history of pancreatitis. The patient did not present a juvenile form of chronic pancreatitis. One of the episodes was serious, with 3.5 months of hospitalisation, and so ultrasound-guided percutaneous drainage of abdominal fluid collection was necessary. During the third hospital-

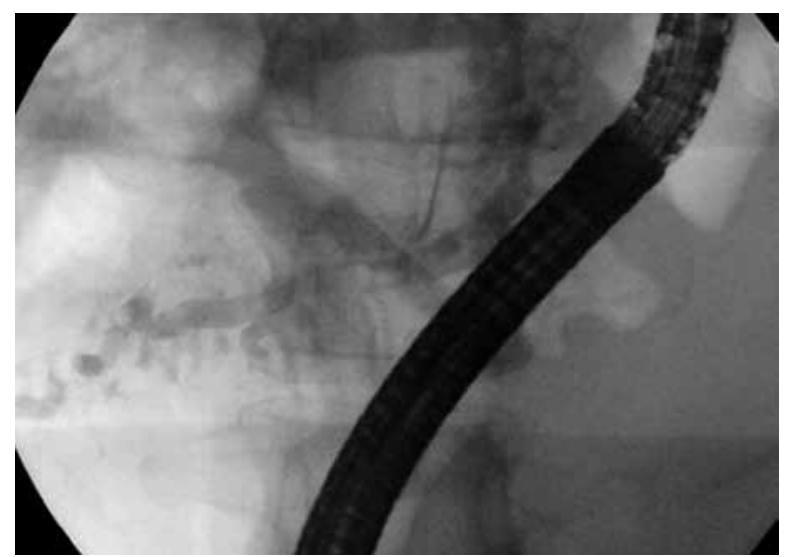

Figure 1. Endoscopic retrograde cholangiopancreatography demonstrating that dilated dorsal duct of Santorini is the major duct to the tail of the pancreas and that it does not communicate with the ventral duct of Wirsung in the pancreatic head (complete pancreas divisum) isation, ERCP was carried out and diagnosis of complete pancreas divisum was made (Figure 1). In this endoscopic procedure a minor duodenal papilla was incised and a plastic stent was inserted into the dorsal pancreatic duct. When the ventral duct was cannulised there was no flow of contrast with the characteristic appearance of pancreas divisum. Thereafter, during a 2-year period, the patient was hospitalised 6 times because of recurrent episodes of pancreatitis with several endoscopic interventions, including exchange of stents and removal of pancreatic duct stones. Throughout this period of time, in the image diagnostics, gradual evolution of the pancreatic parenchyma lesions was observed into a clinical picture of chronic pancreatitis, with the formation of pancreatic head mass and dilatation of the pancreatic duct up to $10 \mathrm{~mm}$, duct calcifications and stones. Simultaneously, the patient's requirement of analgesics increased and a slow decrease of the patient's body weight was seen. Total hospitalisation time since diagnosis was 21 weeks. At this point, because of a lack of improvement in the endoscopic and medical treatments, after 36 months of symptoms, the 42-year-old patient was scheduled for elective surgery. The patient's description of pain before surgery using a visual analogue pain scale was 8 , and moderate use of narcotics was necessary 3-5 times per week. Outpatient follow-up was performed every week during the first month after surgery, and then every 3 months postoperatively.

Local resection of the pancreatic head combined with lateral pancreaticojejunostomy was done under general anaesthesia, with no intra- or postoperative complications. The dorsal (Santorini) duct was longitudinally open in the neck, body and tail of the pancreas and subsequently drained into the jejunal loop. Whereas inflamed pancreatic parenchyma anterior to the ventral (Wirsung) duct was removed (slice by slice; altogether about $7 \mathrm{~g}$ of tissue), and a portion of the pancreatic head and uncinate process which was cored out was also drained by the same Roux-en-Y limb (Figure 2). The patient was discharged from hospital on the $7^{\text {th }}$ postoperative day. A pathological report of the resection specimen of the (fragmentised) portion of the pancreatic head confirmed diagnosis of chronic pancreatitis. The patient fully recovered and returned to her job within 3 weeks. Two years after the operation the patient was briefly hospitalised and treated medically because of exacerbation of pancreatitis due to alcohol abuse. Three years after the operation a new onset of diabetes mellitus was recognised and treated with mild doses of insulin. After 72 months of follow-up, the patient demonstrates abnormal glucose metabolism and requires enzyme supplementation. At present the pa- 
tient and is under the control of the outpatient clinic and, most importantly, she is free of pain.

\section{Discussion}

Pancreas divisum represents a congenital anomaly in the pancreatic ducts, leading to obstructive pancreatitis. Endoscopic/surgical sphincterotomy of the minor duct remains an unproven therapy [13]. Moreover, there is recent evidence based on a randomised controlled trial that surgical drainage of the pancreatic duct is more effective than endoscopic treatment in patients with obstruction of the pancreatic duct due to chronic pancreatitis [14]. Various surgical techniques have been used to decrease resistance to the flow of bile or pancreatic juice and to facilitate the passage of material from the pancreatic ducts both in children and in adults with symptomatic PD: open surgical accessory sphincteroplasty (and distal pancreatectomy), lateral pancreaticojejunostomy, duodenum preserving pancreatic head resection or even pylorus-preserving pancreatoduodenectomy [15-17].

Ten per cent of patients operated on for PD-associated chronic pancreatitis required reoperation with pancreatic resection for failure of lateral pancreaticojejunostomy, to improve chronic pain [18]. Failure of the drainage procedure alone in patients with PD may be related to the following factors: inadequate drainage of the gland head, failure to drain small ducts, and perineural inflammation. The obstructive form of chronic pancreatitis is most easily attributed to PD when structural changes are confined to the dorsal pancreas. Intraductal hypertension is known to play a role in the pathogenesis of pain, and may also have a role in fibrogenesis and glandular damage in patients with obstructive CP.

Whether PD causes pancreatitis is a controversial issue [13]. Gelrud et al. found that 12 patients with PD and recurrent acute pancreatitis had a decrease in cystic fibrosis gene product (CFTR) function [2]. It is speculated that PD could be an incidental finding, or alternatively, in combination with CFTR dysfunction, may be important in the genesis of pancreatitis. Proponents of PD as a cause of pancreatitis focus on the latter half of the statement rather than emphasising that PD is a common variant of pancreatic ductal anatomy and a likely innocent bystander in patients with pancreatitis and PD. In order to identify genetic abnormalities that cause pancreatitis, patients should have genetic analysis of CFTR, HP, SPINK1 or PRSS1 mutations. However this is not routine diagnostic practice.

Another argument to supplement the drainage of the pancreatic duct system with a resection of the pancreatic parenchyma is a possible incidence of intraductal (papillary mucinous) neoplasia [19].

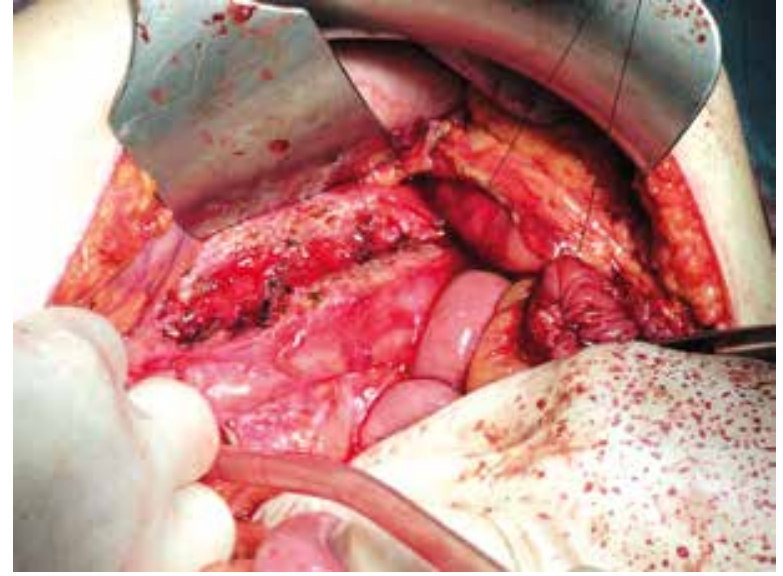

Figure 2. View of the operative field after opening the dorsal duct and coring out of the pancreatic head and uncinate process

In PD, pancreatitis is caused by stenosis at the accessory papilla of Santorini. There may be progression from recurrent acute pancreatitis to irreversible fibrosis in some cases. Sphincteroplasty is effective for recurrent acute pancreatitis, but ductal drainage or resection becomes necessary once chronic pancreatitis is established [8]. Combined duct drainage and/or resection procedures were offered for causal treatment of this form of pancreatitis because of the underlying duct anomalies and pathology in the pancreatic head [9].

Several studies indicate that pancreatitis associated with PD does not develop exclusively in the dorsal duct system, but a ventral duct component is present in up to $12 \%$ and is the only duct affected in over $4 \%$ of cases with PD [13]. Out of the 74 patients with PD and recurrent pancreatitis, histological evidence of inflammation and/or fibrosis was observed in tissues drained by the ventral and dorsal duct systems in $41 \%$ and $58 \%$ of cases, respectively [20]. Local resection of the head of the pancreas combined with longitudinal pancreaticojejunostomy successfully decompresses or resects the main duct of Santorini, the duct to the uncinate and tributary ducts in the head of the pancreas, and decompresses the main duct in the neck, body and tail of the pancreas [21].

Local resection of the pancreatic head combined with lateral pancreaticojejunostomy (Frey procedure) seems to be a highly versatile procedure for chronic pancreatitis, including the form caused by PD. This report provides, to the best of our knowledge, the first description of the Frey procedure successfully applied for the treatment of pancreatitis caused by pancreas divisum in an adult after failure of endoscopic interventions. 
We performed the procedure in September 2006, and the patient has long term follow-up of nearly 8 years now. Recently 6 cases of the Frey procedure for this rare indication has been published, confirming our initial experience [22].

\section{Conclusions}

Local resection of the pancreatic head combined with lateral pancreaticojejunostomy (Frey procedure) offers a favourable long-term outcome after failure of endoscopic papillotomy and duct stenting for pancreatitis associated with pancreas divisum. Although this is a rare form of pancreatitis, further efforts should be undertaken to demonstrate the value of this surgical approach in a larger number of patients.

\section{References}

1. Lerner A, Branski D, Lebenthal E. Pancreatic diseases in children. Pediatr Clin North Am 1996; 43: 125-56.

2. Gelrud A, Sheth S, Banerjee S, et al. Analysis of cystic fibrosis gene product (CFTR) function in patients with pancreas divisum and recurrent acute pancreatitis. Am J Gastroenterol 2004; 99: 1557-62.

3. Gregg JA. Pancreas divisum: its association with pancreatitis. Am J Surg 1977; 134: 539-43.

4. Cotton PB. Congenital anomaly of pancreas divisum as cause of obstructive pain and pancreatitis. Gut 1980; 21: 105-14.

5. Smanio T. Proposed nomenclature and classification of the human pancreatic ducts and duodenal papillae. Study based on 200 post mortems. Int Surg 1969; 52: 125-41.

6. Keith RG, Shapero TF, Saibil FG, Moore TL. Dorsal duct sphincterotomy is effective long-term treatment of acute pancreatitis associated with pancreas divisum. Surgery 1989; 106: 660-6.

7. Rusnak CH, Hosie RT, Kuechler PM, et al. Pancreatitis associated with pancreas divisum: results of surgical intervention. Am J Surg 1988; 155: 641-3.

8. Warshaw AL, Richter JM, Schapiro RH. The cause and treatment of pancreatitis associated with pancreas divisum. Ann Surg 1983; 198: 443-52.

9. Schlosser W, Rau BM, Poch B, Beger HG. Surgical treatment of pancreas divisum causing chronic pancreatitis: the outcome benefits of duodenum-preserving pancreatic head resection. J Gastrointest Surg 2005; 9: 710-5.

10. Frey CF, Smith GJ. Description and rationale of a new operation for chronic pancreatitis. Pancreas 1987; 2: 701-7.

11. Ho HS, Frey CF. The Frey procedure: local resection of pancreatic head combined with lateral pancreaticojejunostomy. Arch Surg 2001; 136: 1353-8.

12. Bell RH Jr. Current surgical management of chronic pancreatitis. J Gastrointest Surg 2005; 9: 144-54.

13. Fogel EL, Toth TG, Lehman GA, et al. Does endoscopic therapy favorably affect the outcome of patients who have recurrent acute pancreatitis and pancreas divisum? Pancreas 2007; 34: 21-45.
14. Cahen DL, Gouma DJ, Nio Y, et al. Endoscopic versus surgical drainage of the pancreatic duct in chronic pancreatitis. N Engl J Med 2007; 356: 676-84.

15. Neblett WW 3rd, O'Neill JA Jr. Surgical management of recurrent pancreatitis in children with pancreas divisum. Ann Surg 2000; 231: 899-908.

16. Varshney S, Johnson CD. Surgery for pancreas divisum. Ann R Coll Surg Engl 2002; 84: 166-9.

17. Hsu JT, Chen HM, Jan YY, et al. Chronic pancreatitis with pancreas divisum treated with pylorus-preserving pancreatoduodenectomy: a case report. Hepatogastroenterology 2001; 48: 1770-1.

18. Schnelldorfer T, Adams DB. Outcome after lateral pancreaticojejunostomy in patients with chronic pancreatitis associated with pancreas divisum. Am Surg 2003; 69: 1041-4.

19. Talbot ML, Foulis AK, Imrie CW. Total dorsal pancreatectomy for intraductal papillary mucinous neoplasm in a patient with pancreas divisum. Pancreatology 2005; 5: 285-8.

20. Madura JA, Madura JA 2nd, Sherman S, Lehman GA. Surgical sphincteroplasty in 446 patients. Arch Surg 2005; 140: 504-11.

21. Frey CF, Mayer KL. Comparison of local resection of the head of the pancreas combined with longitudinal pancreaticojejunostomy (Frey procedure) and duodenum-preserving resection of the pancreatic head (Beger procedure). World I Surg 2003; 27: 1217-30

22. Pappas SG, Pilgrim CHC, Keim R, et al. The Frey procedure for chronic pancreatitis secondary to pancreas divisum. JAMA Surg 2013; 148: 1057-62.

Received: $\quad 30.03 .2011$

Accepted: 15.05 .2014 